\title{
A Review on Recent Computational Methods for Predicting Noncoding RNAs
}

\author{
Yi Zhang, ${ }^{1}$ Haiyun Huang, ${ }^{1}$ Dahan Zhang, ${ }^{2}$ Jing Qiu, ${ }^{3}$ Jiasheng Yang, \\ Kejing Wang, ${ }^{1}$ Lijuan Zhu, ${ }^{1}$ Jingjing Fan, ${ }^{1}$ and Jialiang Yang ${ }^{5}$
}

\author{
${ }^{1}$ Department of Mathematics and Information Retrieval of Library and Hebei Laboratory of Pharmaceutic Molecular Chemistry, \\ Hebei University of Science and Technology, Shijiazhuang, Hebei 050018, China \\ ${ }^{2}$ College of Life Science and Technology, Huazhong Agricultural University, Wuhan, Hubei 430070, China \\ ${ }^{3}$ Department of Network Engineering, School of Information Science and Engineering, \\ Hebei University of Science and Technology, Shijiazhuang 050018, China \\ ${ }^{4}$ Department of Civil and Environmental Engineering, National University of Singapore, Singapore 117576 \\ ${ }^{5}$ School of Mathematics and Information Science, Henan Polytechnic University, Henan 454000, China
}

Correspondence should be addressed to Yi Zhang; zhaqi1972@163.com and Jialiang Yang; jialiang.yang@mssm.edu

Received 29 November 2016; Revised 6 February 2017; Accepted 15 February 2017; Published 3 May 2017

Academic Editor: Ernesto Picardi

Copyright (c) 2017 Yi Zhang et al. This is an open access article distributed under the Creative Commons Attribution License, which permits unrestricted use, distribution, and reproduction in any medium, provided the original work is properly cited.

\begin{abstract}
Noncoding RNAs (ncRNAs) play important roles in various cellular activities and diseases. In this paper, we presented a comprehensive review on computational methods for ncRNA prediction, which are generally grouped into four categories: (1) homology-based methods, that is, comparative methods involving evolutionarily conserved RNA sequences and structures, (2) de novo methods using RNA sequence and structure features, (3) transcriptional sequencing and assembling based methods, that is, methods designed for single and pair-ended reads generated from next-generation RNA sequencing, and (4) RNA family specific methods, for example, methods specific for microRNAs and long noncoding RNAs. In the end, we summarized the advantages and limitations of these methods and pointed out a few possible future directions for ncRNA prediction. In conclusion, many computational methods have been demonstrated to be effective in predicting ncRNAs for further experimental validation. They are critical in reducing the huge number of potential ncRNAs and pointing the community to high confidence candidates. In the future, high efficient mapping technology and more intrinsic sequence features (e.g., motif and $k$-mer frequencies) and structure features (e.g., minimum free energy, conserved stem-loop, or graph structures) are suggested to be combined with the next- and third-generation sequencing platforms to improve ncRNA prediction.
\end{abstract}

\section{Background}

A noncoding RNA (ncRNA) is a functional RNA that is transcribed from a DNA but does not encode a protein. According to transcriptomic and bioinformatics studies, there are thousands of ncRNAs classified into different categories based on their functions and lengths including transfer RNA (tRNA), ribosomal RNA (rRNA), microRNA (miRNA), and long ncRNA (lncRNA) to name a few [1-3].

These ncRNAs play important roles in various cellular processes. For example, rRNA catalyzes the peptide bond formation between amino acids in translation process [4], miRNA is important in transcription process and performs posttranscriptional regulation of gene expression [5], and lncRNA plays critical diverse roles in X inactivation, imprinting, and regulation of epigenetic marks and gene expression [6-8]. In addition, they also exhibit enormous importance in connection with various diseases. For example, the miR-17-92 cluster functions as oncogenes while the miR-15a-miR-16-1 cluster functions as tumour suppressors [9]. ANRIL, one type of lncRNA, is related to coronary disease, type II diabetes, and intracranial aneurysm [10]. The readers are referred to a review by Esteller [11] and Chen et al. [12] for more information about specific correlations between ncRNAs and human diseases. Specifically, Esteller [11] provides a review on the relationship between dysfunctions of ncRNAs including 


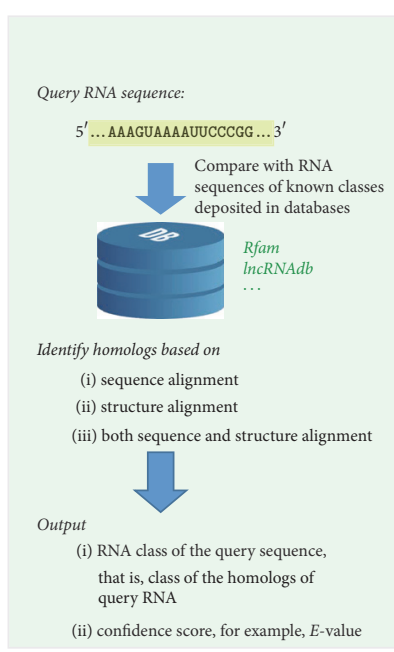

(a)

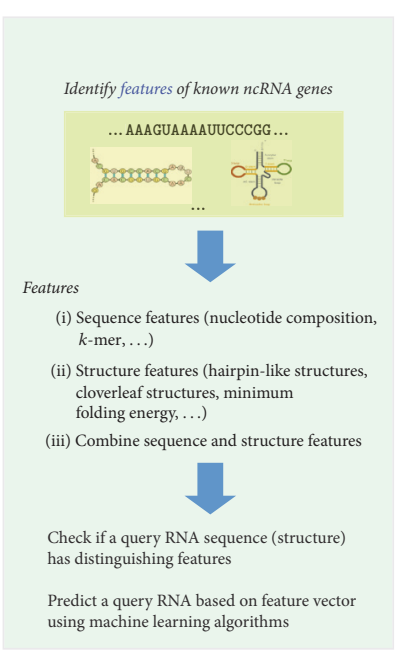

(b)

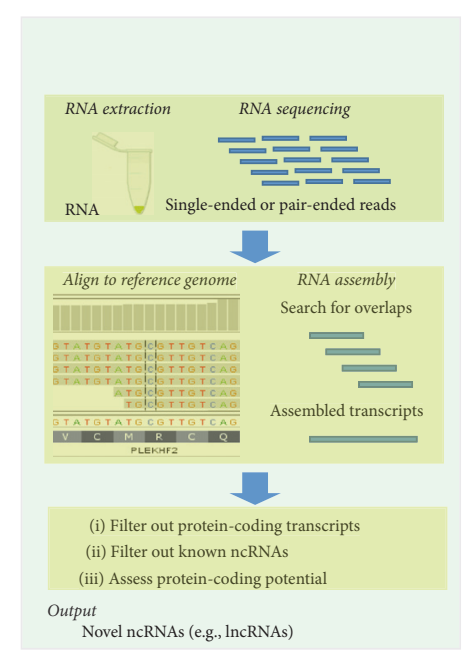

(c)

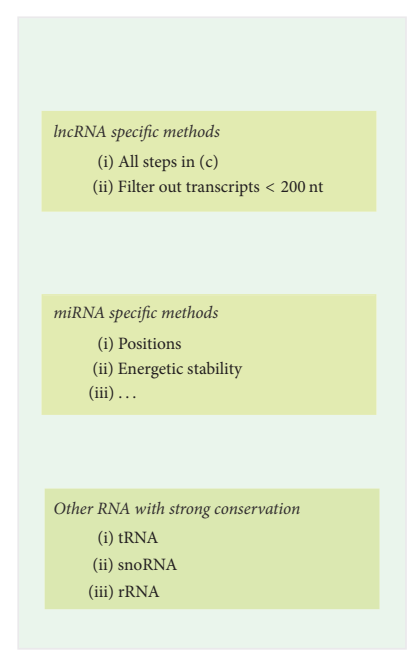

(d)

FIGURE 1: Four popular categories of computational methods in predicating ncRNAs. (a) Homology-based methods, which compare a query RNA with known ncRNAs deposited in databases based on sequence or structure alignment; (b) de novo methods, which predict ncRNA from primary sequences or structure based on general principles that govern ncRNA folding or statistical tendencies of $k$-mer features; (c) transcriptional sequencing and assembling based methods, which utilize next-generation sequencing and transcriptome data; and (d) RNA family specific methods, which predict specific ncRNA classes.

miRNA, PIWI-interacting RNAs (piRNAs), small nucleolar RNAs (snoRNAs), transcribed ultraconserved regions (TUCRs), and large intergenic noncoding RNAs (lincRNAs) and a few diseases including tumorigenesis and neurological, cardiovascular, developmental, and other diseases. Chen et al. [12] discussed the roles of lncRNAs in critical biological processes and human diseases like various cancers, diabetes, and AIDS.

Due to the important roles of ncRNAs in cellular processes and disease development, many experimental and bioinformatics methods have been developed to predict ncRNAs and their functions. As for experimental methods, enzymatic and chemical RNA sequencing, parallel cloning of ncRNAs by specialized cDNA libraries, microarray analysis, and genomic SELEX are among the most popular ones. The readers are referred to a review paper for the details of these methods [13]. However, the experimental methods are expensive and time-consuming, and thus hundreds of computational methods have also been developed to prioritize highly confident ncRNA candidates for further experimental validation. In this paper, we present a comprehensive review on these computational methods. We are fully aware that there have already been several review articles on this hot topic [14-17]. However, they either focus on a specific ncRNA category or have been outdated and could not present a panoramic view of the field.

\section{Main Text}

Generally speaking, there are three major categories of computational methods in predicting ncRNAs, namely, (1) homology-based methods involving evolutionarily conserved RNA sequences and structures, (2) de novo methods using RNA sequence and structure features, and (3) transcriptional sequencing and assembling based methods, according to chronological order of their occurrences. Since miRNA and lncRNA have very specific methods, we reviewed them separately and called these methods RNA family specific methods (Figure 1).

2.1. Homology-Based Methods. As probably the earliest ncRNA prediction methods, homology-based methods assume that sequence or structure similar RNAs are evolved from a common ancestor and thus share function similarities $[18,19]$. Given a query RNA, these methods usually compare it with known ncRNAs deposited in databases based on sequence or structure alignment. The RNA is predicted to be in a specific ncRNA family if it has sufficient similarity with known ncRNAs in that family (Figure 1(a)). There are a number of ncRNA databases. For example, 2,474 structural families of ncRNAs were cataloged in the database Rfam (version 12.1, April 2016) [20]. We listed a few popular homology-based methods in Table 1, which are further classified into sequence-based methods, structure-based methods, and hybrid methods.

2.1.1. Sequence-Based Methods. These methods rely purely on sequence conservations inferred by alignment methods like BLAST [18] and BLAT [21]. They first identify short (gapped) matches called seeds [22] between the query ncRNA and any ncRNA in the database, which are then expanded in both directions to form high-scoring segment pair (HSPs). The statistical significance of a HSP or the joining of several HSPs is evaluated by expected value (called $E$-value). The query ncRNA is classified into the family containing the ncRNA with the lowest $E$-value. 


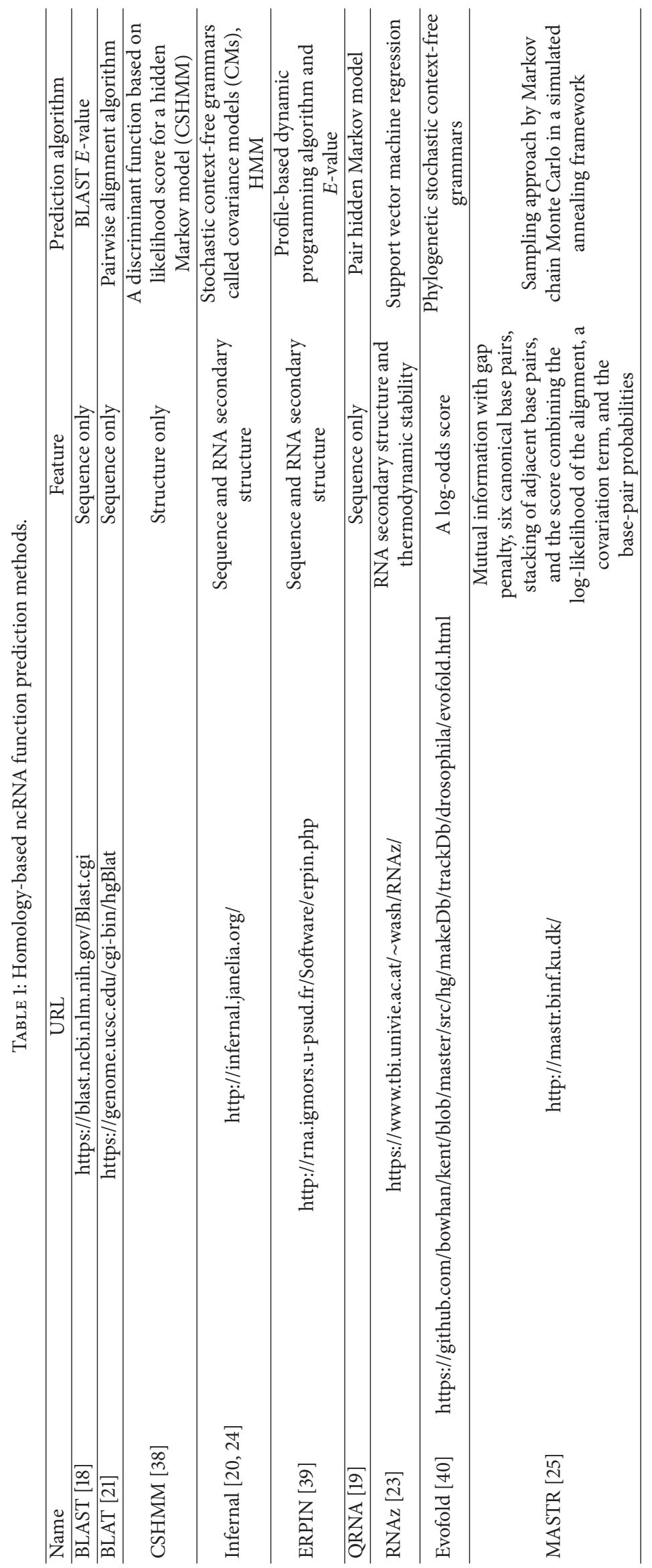


2.1.2. Structure-Based Methods. Sequence-based methods are usually very fast. However, it is commonly believed that ncRNAs are less conserved in sequence. Thus, another category of homology-based methods is introduced based on structure conservations. Instead of sequence alignment, these methods use RNA secondary structure alignment to measure RNA similarity. Popular methods include QRNA [19] and RNAz [23]. Specifically, QRNA compares query RNA with known RNAs using "three probabilistic pairgrammars: a pair stochastic context-free grammar modeling alignments constrained by structural RNA evolution, a pair hidden Markov model modeling alignments constrained by coding sequence evolution, and a pair hidden Markov model modeling a null hypothesis of position-independent evolution" [19], whereas RNAz compares RNAs based on conserved secondary structure and thermodynamic stability [23].

2.1.3. Hybrid Methods. A more robust RNA similarity measure was obtained by incorporating both sequence and structure information. For example, Infernal [24] uses covariance models, which score a combination of sequence consensus and RNA secondary structure consensus to predict ncRNAs homologous to ncRNA families in Rfam [20, 24]. MASTR [25] makes use of simulated annealing method to perform sequence alignment and structural alignment simultaneously.

Though homology-based methods have been extensively used due to their advantages in speed, however, they have a few limitations. First, they compare the query RNA with known ncRNA families and thus are incapable of predicting new ncRNA families. Second, they rely on sequence or structure conservations and thus are inapplicable to predict ncRNAs lacking conservation in sequence and structure. As a result, de novo methods are proposed to solve such dilemma.

2.2. De Novo Methods Using RNA Sequence and Structure Features. Unlike homology methods which require the information of RNAs similar (or homologous) to the query RNA, de novo methods predict ncRNA from primary sequences or structure based on general principles that govern ncRNA folding energetics and/or statistical tendencies of $k$-mer features that native ncRNA sequences and structures acquire (Figure 1(b)). Based on the source of common features, de novo methods can be divided into sequence feature based methods which only use sequence features, structure feature methods, and hybrid feature methods which use both features.

2.2.1. Sequence Feature Based Methods. One important feature for sequence-based de novo methods is nucleotide composition, which applies for identifying ncRNAs in species with nucleotide compositional biases. For example, by calculating the GC content, Wang et al. identified ncRNA genes with stable secondary structure in an AT-rich extreme hyperthermophile [26]. Another commonly used nucleotide composition is $k$-mer (nucleotide sequence of length $k$ ) frequencies. Methods in this category exploit the finding that the frequencies of many $k$-mers for ncRNAs in a specific family usually share similar probability distribution. Thus, new ncRNAs can be predicted based on the distribution of their $k$-mer frequencies. For example, Panwar et al. used the trinucleotide composition (i.e., 3-mer) to predict ncRNA by a support vector machine (SVM) based algorithm [27]. Sun et al. proposed Coding-Non-Coding Index (CNCI), by profiling adjoining nucleotide triplets (i.e., 6-mer) to effectively distinguish protein-coding and noncoding sequences independent of known annotations [28]. In addition, Li et al. developed an algorithm named PLEK to discriminate lncRNAs from mRNAs based on a combination of 1 to 5 mers [29].

Since a single type of sequence feature might be insufficient in effectively identifying ncRNAs, other features have also been proposed in conjunction with nucleotide composition. We summarized a few popular sequence feature based de novo ncRNA identification methods in Table 2. For example, CONC [30] incorporates a few types of features including sequence length, nucleotide composition, and reading frame to characterize ncRNAs. CPC [31] combines the longest reading frame in the three forward frames, logodds score, coverage of the predicted ORF, and integrity of the predicted ORF, to identify ncRNAs.

2.2.2. Structure Feature Based Methods. The secondary structures of some kinds of functional RNA are more conserved than their primary sequences [32]. For example, miRNA precursors share common hairpin-like structures and tRNAs share cloverleaf structures. The structure with (or around) the minimum folding energy (MFE) is usually regarded as the most possible fold structure of an RNA. Thus, MFE is extensively used to predict secondary structure of ncRNA sequences. Popular MFE-based methods include RNAfold [33], Mfold [34], and Afold [35]. RNAfold calculates MFE by assigning free energies to both loops and stems, whereas Mfold only assigns free energies to loops. Afold improves the speed in evaluating all possible internal loops by an algorithm constructing sets of conditionally optimal multibranch loop free (MLF) structures. However, it is generally insufficient to use MFE alone for the detection of ncRNAs since different secondary structures of a given RNA sequence may have very similar MFE [36]. As a result, more structure features like thermodynamic stability are also employed in predicting ncRNA [37].

2.2.3. Hybrid Feature Based Methods. As a trend, more and more de novo methods tend to combine both RNA sequence and RNA structure to improve the sensitivity and specificity in predicting ncRNAs.

For example, Gupta et al. developed a new algorithm ptRNApred to identify and classify posttranscriptional RNA with dinucleotide properties of sequence and secondary structure feature, for example, numbers of loops, bulges, and hairpins or the frequency of nucleotides involved in substructures [45]. It can predict ptRNA-subclasses in eukaryotes including snRNA, snoRNA, RNase P, RNase MRP, Y RNA, and telomerase RNA. We summarized popular de novo ncRNA prediction methods using RNA sequence and structure features in Table 3. For a better view, we also plotted some popular de novo methods and their prediction algorithms in 


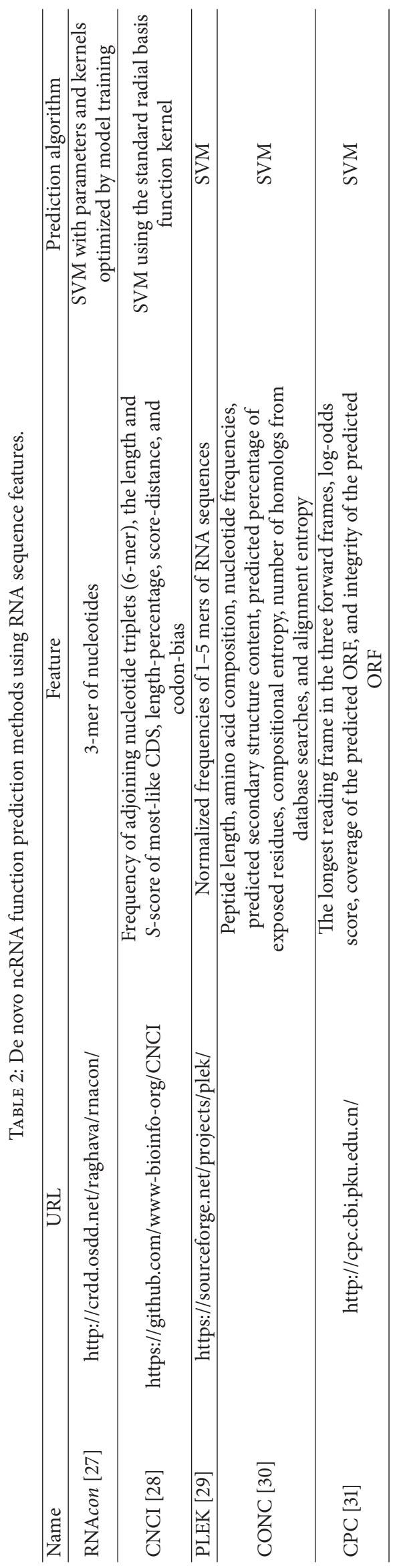




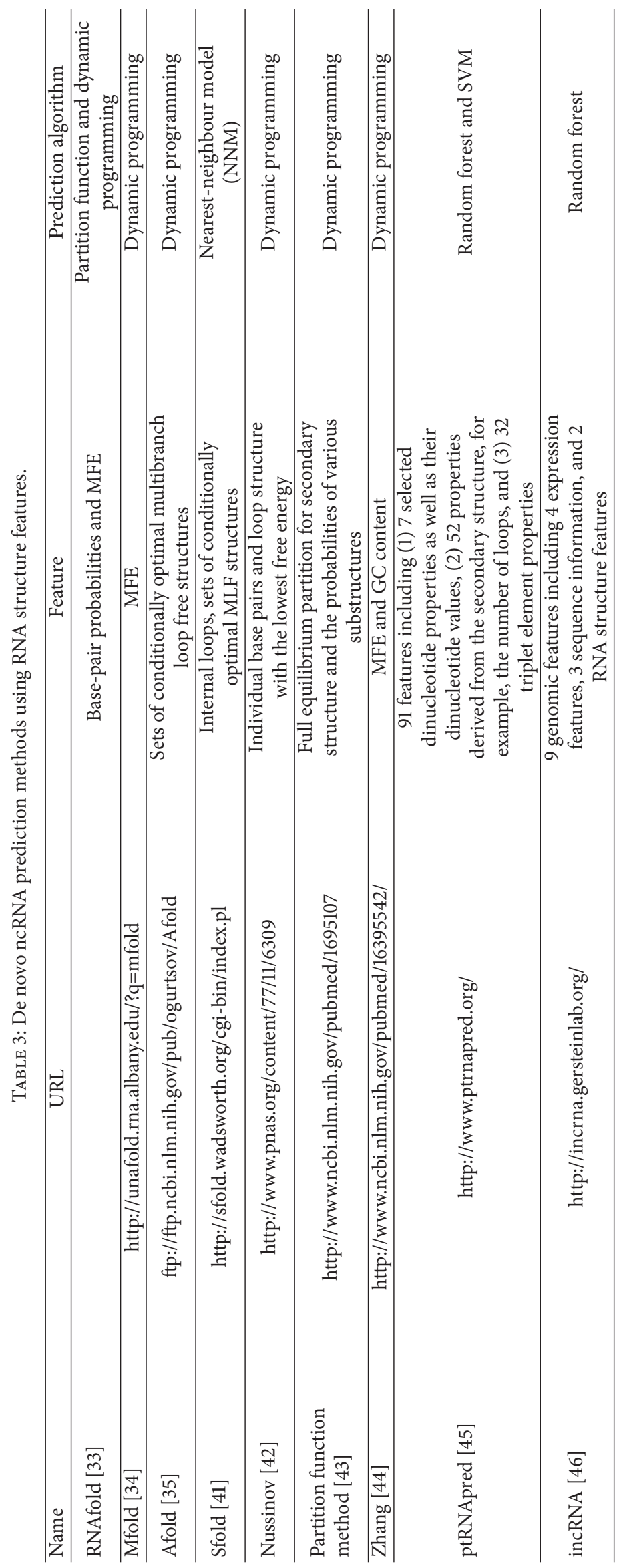




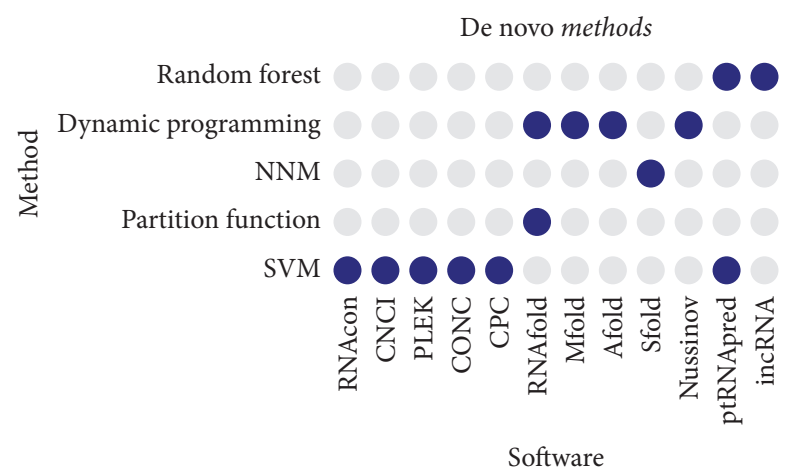

FIgURE 2: Popular de novo methods and the statistical algorithms applied.

Figure 2. Support vector machine (SVM) is probably the most frequently used method for de novo ncRNA prediction.

De novo methods are capable of predicting new ncRNA families and classifying ncRNAs lacking conservation with existing ones. They usually have higher sensitivity and lower specificity than homology-based methods. However, this kind of methods depends largely on the features extracted. With the enrichment of biological, chemical, and dynamic knowledge of ncRNA, there might be some further informative features to be extracted, which will greatly benefit de novo ncRNA prediction $[46,68]$.

2.3. Transcriptional Sequencing and Assembling Based Methods. More recently, with the advances in next-generation sequencing (NGS), especially RNA sequencing (RNA-seq) techniques, more and more transcriptome data are available, which have been utilized to discover novel ncRNAs. A general workflow of transcriptional sequencing and assembling based ncRNA prediction method is described in Figure 1(c). Different from homology-based and de novo methods which require specific RNA sequences, methods in this category usually start from raw single-ended or pair-ended reads. The reads are then mapped into a reference genome and the mapped reads are assembled into transcripts based on overlapping information. After removing protein-coding RNA and known ncRNA transcripts, the remaining transcripts are further assessed for protein-coding potential and novel ncRNAs are reported if the potential is low.

In practice, RNA-seq data are usually combined with other features and methods including tilling array [47], graph-kernel SVM [49], structure features and common motifs [69], differential gene expression (DGE) data [48], and exon array [50] to predict specific ncRNAs. For example, tiling array [47] is used to scan the long and macro non-protein-coding RNAs related to cell-cycle, p53, and STAT3 pathways. DGE is used for discovering novel polyA+noncoding transcripts within human genome [48]. BlockClust [49] tries to predict the ncRNA modified after its transcription by combining the sequence and secondary structure information with a graph-kernel SVM, whose novel thinking lies in a new strategy to formulate expression profiles in compact discrete structures using fast graph-kernel techniques. We summarized some popular sequencing and assembling based ncRNA predication algorithms in Table 4 .

As an advantage over homology-based methods and de novo methods, RNA-seq based methods can directly sequence coding and noncoding RNA transcripts with high sensitivity and low false positive rate. It can especially detect new scripts and alternative splicing. However, sometimes it is difficult to tell ncRNAs from protein-coding RNAs and thus other features like sequence conservation [53], deciphering abstract graphical representation [49], designing exon probes [50], finer terminal stem-loop feature [51], or $k$-mer frequency [52] are often utilized together with RNAseq analysis to infer ncRNAs. In this sense, one may regard the RNA-seq technology as a platform rather than a certain method.

2.4. RNA Family Specific Methods. Since miRNA and lncRNA are two special and important ncRNAs, we reviewed a few computational methods related to them separately (Figure $1(\mathrm{~d}))$.

2.4.1. miRNA Specific Methods. miRNAs are very short in length, usually around $22 \mathrm{nt}$. The short length and relatively low conservation of pre-miRNA sequences restrict the usage of sequence-based methods in identifying miRNAs. Fortunately, it is known that miRNAs are mostly derived from regions of RNA transcripts that fold back on themselves to form short hairpins, which make this RNA relatively conserved in secondary structure. Thus, a few methods exploit more secondary features for new miRNA gene detection instances. For example, as a homology-based method, miRAlign employs sequence alignment, secondary structure alignment, and miRNA's position on the stem-loop structure to identify RNA homologs. It has higher sensitivity and comparable specificity than other homology-based methods [70]. MiPred adopts the local contiguous structure sequence composition, MFE, and $P$ value of randomization test to predict miRNA precursor with a random forest algorithm [54]. We summarized popular methods for predicting miRNA in Table 5.

2.4.2. IncRNA Specific Methods. Long noncoding RNAs (lncRNAs) are ncRNAs longer than $200 \mathrm{nt}$, including long intronic noncoding RNA and intergenic noncoding RNA. lncRNAs are believed to regulate gene expression through changing chromatin state and correlate with cancer pathogenesis and various clinical traits [63-66, 71]. In fact, lncRNA prediction is a very challenging task, because many lncRNAs exhibit low sequence and structure conservation; moreover, they are often capped and spliced. Some databases like lncRNAdb [72] provide comprehensive annotations of specific lncRNAs, for example, eukaryotic lncRNAs. A general flow to identify lncRNA is as follows: first the transcriptome data are annotated and the protein-coding sequences are filtered; then sequences shorter than $200 \mathrm{nt}$ are removed and the remaining ones are viewed as candidate lncRNAs [63]; finally, the candidate lncRNAs are evaluated based on features like secondary structures $[73,74]$, protein-coding ability [28, 29], conserved splicing sites [75], DGE+RNA-seq, conserved 


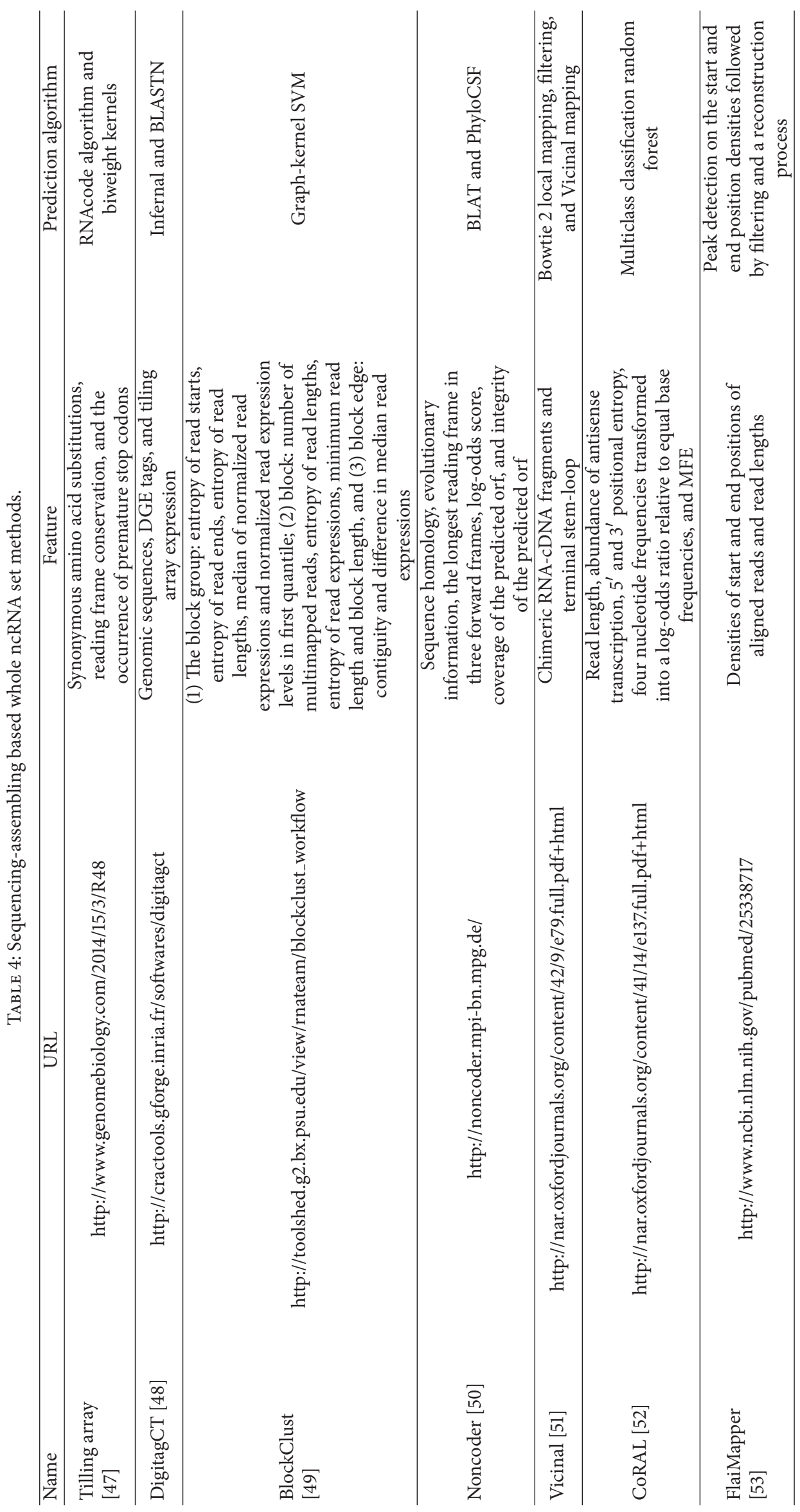




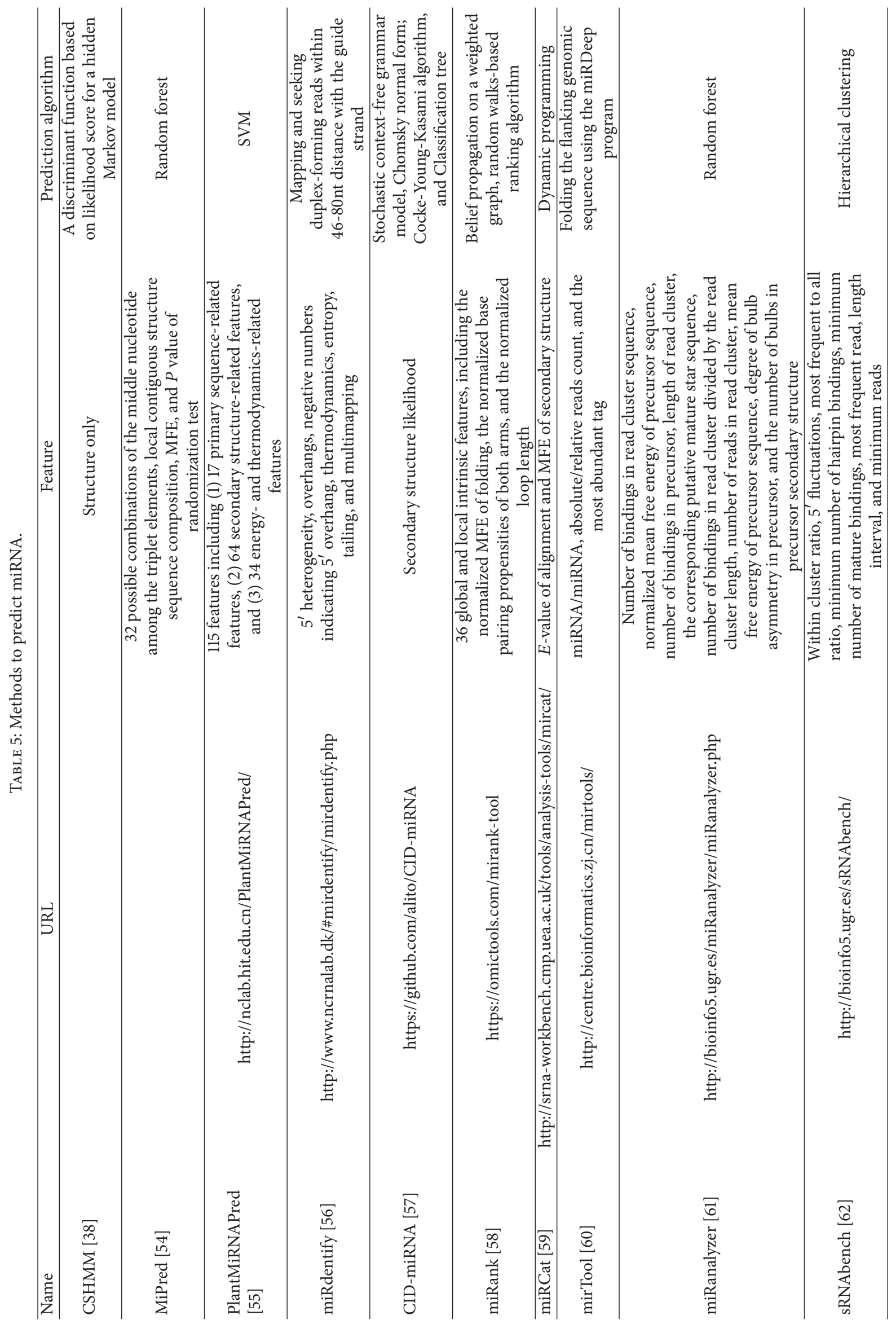


TABLE 6: Methods to predict lncRNAs.

\begin{tabular}{lcc}
\hline Name & Feature & Prediction algorithm \\
\hline $\begin{array}{l}\text { Estimating lincRNome size for } \\
\text { human [63] }\end{array}$ & $\begin{array}{r}\text { lincRNA numbers validated experimentally in human and mouse, } \\
\text { and their overlap lincRNA number }\end{array}$ & System of nonlinear equations \\
\hline Classifying human lncRNA [64] & $\begin{array}{r}\text { RNA sequence-structure patterns (RSSP) describing 42 highly } \\
\text { structured families, motif binding sites extracted as 1314 } \\
\text { Position-Weight Matrices (PWMs), all } k \text {-words of length } \\
k=2,3,4,5,6,7,8 \text {, the sequence complexity }\end{array}$ & $\begin{array}{c}\text { Classifying human lncRNA by } \\
\text { being able (or disable) to bind } \\
\text { the polycomb repressive complex } \\
\text { (PRC2), SVM with linear kernel }\end{array}$ \\
\hline $\begin{array}{l}\text { Identify, classify, and localize } \\
\text { maize lncRNAs [65] }\end{array}$ & $\begin{array}{c}\text { Transcript length, open reading frame (ORF) size, and homology } \\
\text { with known proteins }\end{array}$ & SVM \\
\hline $\begin{array}{l}\text { The GENCODE v7 catalog of } \\
\text { human lncRNA [66] }\end{array}$ & $\begin{array}{c}\text { Lack of homology with known proteins, no reasonable-sized open } \\
\text { reading frame (ORF), and no high conservation, confirmed by } \\
\text { PhyloCSF through the majority of exons conserved promoters }\end{array}$ & $\begin{array}{c}\text { Manual annotation and pattern } \\
\text { recognition }\end{array}$ \\
\hline $\begin{array}{l}\text { Highly conserved large } \\
\text { noncoding RNAs [67] }\end{array}$ & Chromatin signatures "K4-K36" domain & $\begin{array}{c}\text { Maximum CSF score observed } \\
\text { across the entire genomic locus }\end{array}$ \\
\hline
\end{tabular}

promoters [66], and chromatin signatures such as "K4-K36" domain [67], and only those that pass certain significance levels are inferred to be lncRNAs. We summarized popular lncRNA prediction methods in Table 6.

Besides the above two RNA families, some specific classification and prediction methods have been developed for ncRNAs with strong conservation information, for example, tRNA [76-78], snoRNA [79-81], and rRNA [82]. Recently, the largest ncRNA set, piRNA, can be predicted by an improved Fisher algorithm with 1364-D vectors representing RNA sequences $[83,84]$.

\section{Conclusions}

It is very important to predict ncRNAs since they are related to many diseases $[85,86]$. Many ncRNA sequences are stored in databases such as fRNAbd [87], NONCODE [88], and Rfam [20] and grouped into classes based on their structures. The popular software Infernal [24] can predict 2,474 families of ncRNA. However, there are still ncRNAs that cannot be predicted by Infernal, including piRNA, Air, BC200, mature miRNA, gRNA, mRNA-like RNA, BC1 RNA, BM1 RNA, and so on. The major issue is that these ncRNAs lack sequence and structure conservation. To thoroughly predict the ncRNA classes and whole ncRNA set, we need to construct a series of new methods, including extracting new features and developing novel algorithms.

Homology search has become much faster with the development of bioinformatics tools, for example, from SmithWaterman dynamic programming algorithm to BLAST or GMAP [89] based on simplified consecutive $k$-mer match or gapped $k$-mer (also called spaced seeds) techniques [22, 90]. However, these methods are less sensitive in ncRNA identification. On the other hand, de novo algorithms try to retrieve significant intrinsic features from RNA sequences, structures, energy, stability, and even deep-sequencing mapping profile. They use the features to discriminate a certain class of ncRNAs from other RNA sequences. However, de novo algorithms have high false positive rate. At present, how to combine these features and select a proper classifying machine is another hotspot to improve the sensitivity and specificity of ncRNA identification. With the rapid increasing of second- and third-generation sequencing (TGS) data, the information derived from deep-sequencing and singlemolecule long-read sequencing may provide a great opportunity to enhance the efficiency in ncRNA prediction.

In addition, it has become central for understanding biological process by studying RNA globally. However, methods like microarrays and short-read sequencing are incapable of describing the entire RNA molecule from $5^{\prime}$ to $3^{\prime}$ end. Scientists use single-molecule long-read sequencing technology from Pacific Biosciences to sequence the polyadenylated RNA complement for human, without the need for fragmentation or amplification [91]. TGS can get full-length RNA molecules of up to $1.5 \mathrm{~kb}$ with little sequence loss at the $5^{\prime}$ ends. In total, $\sim 14,000$ spliced GENCODE genes of human were identified [91], but $>10 \%$ of the alignments are mapped to unannotated regions; these transcripts are novel noncoding RNAs. Obviously, TGS may give more power to lncRNA discovery.

Finally, in order to assemble and correct long transcripts, one can integrate reads sequenced by five sequencing platforms including Illumina HiSeq, Life Technologies' PGM and Proton, Pacific Biosciences RS, and Roche's 454 [92]. Software programs like TMAP (PGM and Proton), GSRM (454), and GMAP (PacBio) are the best in mapping the sequencing reads to a reference genome. It has been shown that the integration results showed high concordance in both intraplatform and interplatform studies [92]. In addition, the integrated data also performed effectively in analyzing degraded RNA samples. Thus, platform integration is very promising for improvement of RNA-seq as well as ncRNA identification in the future.

\author{
Abbreviations \\ ncRNA: Noncoding RNA \\ RT-PCR: Reverse transcription polymerase chain \\ reaction \\ tRNA: Transfer RNA \\ rRNA: Ribosomal RNA \\ snoRNA: Small nucleolar RNA \\ miRNA: microRNA
}


siRNA: Small interfering RNA

snRNA: Small nuclear RNA

exRNA: Extracellular RNA

piRNA: Piwi-interacting RNA

lncRNA: Long noncoding RNA

Xist: $\quad$ X-inactive specific transcript

HOTAIR: HOX transcript antisense RNA

ceRNA: Competing endogenous RNA

MALAT-1: Metastasis-associated lung adenocarcinoma transcript 1

HSP: High-scoring segment pair

HMM: Hidden Markov model

MFE: $\quad$ Minimum folding energy

SVM: $\quad$ Support vector machine

DGE: Differential gene expression.

\section{Conflicts of Interest}

The authors declare that they have no conflicts of interest.

\section{Authors' Contributions}

Jialiang Yang and Yi Zhang conceived and designed the study. Yi Zhang, Jialiang Yang, Haiyun Huang, Dahan Zhang, Jing Qiu, Jiasheng Yang, Kejing Wang, Lijuan Zhu, and Jingjing Fan were involved in literature mining and summary. Jialiang Yang and Yi Zhang wrote the paper. All authors reviewed and approved the final manuscript. Yi Zhang, Haiyun Huang, and Dahan Zhang contributed equally to this work.

\section{Acknowledgments}

The study was funded by the National Science Foundation (no. 11171088 to Yi Zhang and no. 61300120 to Jing Qiu); the Fundamental Research Funds for the Central Universities (no. 2016BC021 to Dahan Zhang); the Natural Science Foundation of Hebei Province (no. A2015208108 to Yi Zhang); the Educational Commission of Hebei Province on Humanities and Social Sciences (no. SZ16180 to Haiyun Huang); Science and Technology Plan Project of Hebei Province (no. 15210341 to Haiyun Huang); the research project of University Libraries in Hebei Province (201503Z to Haiyun Huang); the Science Fund of the Hebei University of Science and Technology Foundation (no. 2014PT67 to Yi Zhang); and the Hebei Province Foundation for Advanced Talents (no. A201400121 to Yi Zhang).

\section{References}

[1] J. Cheng, P. Kapranov, J. Drenkow et al., "Transcriptional maps of 10 human chromosomes at 5-nucleotide resolution," Science, vol. 308, no. 5725, pp. 1149-1154, 2005.

[2] E. Birney, J. A. Stamatoyannopoulos, A. Dutta et al., "Identification and analysis of functional elements in $1 \%$ of the human genome by the ENCODE pilot project," Nature, vol. 447, no. 7146, pp. 799-816, 2007.

[3] S. Washietl, J. S. Pedersen, J. O. Korbel et al., "Structured RNAs in the ENCODE selected regions of the human genome," Genome Research, vol. 17, no. 6, pp. 852-864, 2007.
[4] P. Nissen, J. Hansen, N. Ban, P. B. Moore, and T. A. Steitz, "The structural basis of ribosome activity in peptide bond synthesis," Science, vol. 289, no. 5481, pp. 920-930, 2000.

[5] K. Chen and N. Rajewsky, "The evolution of gene regulation by transcription factors and microRNAs," Nature Reviews Genetics, vol. 8, no. 2, pp. 93-103, 2007.

[6] R. R. A. Pandey and C. Kanduri, "Transcriptional and posttranscriptional programming by long noncoding RNAs," Progress in Molecular and Subcellular Biology, vol. 51, pp. 1-27, 2011.

[7] J. B. Heo and S. Sung, "Vernalization-mediated epigenetic silencing by a long intronic noncoding RNA," Science, vol. 331, no. 6013, pp. 76-79, 2011.

[8] C. Plessy, G. Pascarella, N. Bertin et al., "Promoter architecture of mouse olfactory receptor genes," Genome Research, vol. 22, no. 3, pp. 486-497, 2012.

[9] G. A. Calin and C. M. Croce, "MicroRNA signatures in human cancers," Nature Reviews Cancer, vol. 6, no. 11, pp. 857-866, 2006.

[10] H. M. Broadbent, J. F. Peden, S. Lorkowski et al., "Susceptibility to coronary artery disease and diabetes is encoded by distinct, tightly linked SNPs in the ANRIL locus on chromosome 9p," Human Molecular Genetics, vol. 17, no. 6, pp. 806-814, 2008.

[11] M. Esteller, "Non-coding RNAs in human disease," Nature Reviews Genetics, vol. 12, no. 12, pp. 861-874, 2011.

[12] X. Chen, C. C. Yan, X. Zhang, and Z. You, "Long non-coding RNAs and complex diseases: from experimental results to computational models," Briefings in Bioinformatics, pp. 1-19, 2016.

[13] A. Hüttenhofer and J. Vogel, "Experimental approaches to identify non-coding RNAs," Nucleic Acids Research, vol. 34, no. 2, pp. 635-646, 2006.

[14] N. E. Ilott and C. P. Ponting, "Predicting long non-coding RNAs using RNA sequencing," Methods, vol. 63, no. 1, pp. 50-59, 2013.

[15] A. Machado-Lima, H. A. del Portillo, and A. M. Durham, "Computational methods in noncoding RNA research," Journal of Mathematical Biology, vol. 56, no. 1-2, pp. 15-49, 2008.

[16] C. Wang, L. Wei, M. Guo, and Q. Zou, "Computational approaches in detecting non- coding RNA," Current Genomics, vol. 14, no. 6, pp. 371-377, 2013.

[17] P. M. Krzyzanowski, E. M. Muro, and M. A. Andrade-Navarro, "Computational approaches to discovering noncoding RNA," Wiley Interdisciplinary Reviews RNA, vol. 3, no. 4, pp. 567-579, 2012.

[18] S. F. Altschul, W. Gish, W. Miller, E. W. Meyers, and D. J. Lipman, "Basic local alignment search tool," Journal of Molecular Biology, vol. 215, no. 3, pp. 403-410, 1990.

[19] E. Rivas and S. R. Eddy, "Noncoding RNA gene detection using comparative sequence analysis," BMC Bioinformatics, vol. 2, article 8, 2001.

[20] P. P. Gardner, J. Daub, J. G. Tate et al., "Rfam: updates to the RNA families database," Nucleic Acids Research, vol. 37, no. 1, pp. D136-D140, 2009.

[21] W. J. Kent, "BLAT-the BLAST-like alignment tool," Genome Research, vol. 12, no. 4, pp. 656-664, 2002.

[22] J. Yang and L. Zhang, "Run probabilities of seed-like patterns and identifying good transition seeds," Journal of Computational Biology, vol. 15, no. 10, pp. 1295-1313, 2008.

[23] S. Washietl, I. L. Hofacker, and P. F. Stadler, "Fast and reliable prediction of noncoding RNAs," Proceedings of the National Academy of Sciences of the United States of America, vol. 102, no. 7, pp. 2454-2459, 2005. 
[24] E. P. Nawrocki, D. L. Kolbe, and S. R. Eddy, "Infernal 1.0: inference of RNA alignments," Bioinformatics, vol. 25, no. 10, pp. 1335-1337, 2009.

[25] S. Lindgreen, P. P. Gardner, and A. Krogh, "MASTR: multiple alignment and structure prediction of non-coding RNAs using simulated annealing," Bioinformatics, vol. 23, no. 24, pp. 33043311, 2007.

[26] X.-J. Wang, J. L. Reyes, N.-H. Chua, and T. Gaasterland, "Prediction and identification of Arabidopsis thaliana microRNAs and their mRNA targets," Genome Biology, vol. 5, no. 9, article R65, 2004.

[27] B. Panwar, A. Arora, and G. P. S. Raghava, "Prediction and classification of ncRNAs using structural information," BMC Genomics, vol. 15, no. 1, article 127, 2014.

[28] L. Sun, H. Luo, D. Bu et al., "Utilizing sequence intrinsic composition to classify protein-coding and long non-coding transcripts," Nucleic Acids Research, vol. 41, no. 17, article e166, 2013.

[29] A. Li, J. Zhang, and Z. Zhou, "PLEK: a tool for predicting long non-coding RNAs and messenger RNAs based on an improved k-mer scheme," BMC Bioinformatics, vol. 15, no. 1, article 311, 2014.

[30] J. Liu, J. Gough, and B. Rost, "Distinguishing protein-coding from non-coding RNAs through support vector machines," PLoS Genetics, vol. 2, no. 4, article e29, 2006.

[31] L. Kong, Y. Zhang, Z.-Q. Ye et al., "CPC: assess the proteincoding potential of transcripts using sequence features and support vector machine," Nucleic Acids Research, vol. 35, no. 2, pp. W345-W349, 2007.

[32] S. Washietl, I. L. Hofacker, M. Lukasser, A. Hüttenhofer, and P. F. Stadler, "Mapping of conserved RNA secondary structures predicts thousands of functional noncoding RNAs in the human genome," Nature Biotechnology, vol. 23, no. 11, pp. 13831390, 2005.

[33] I. L. Hofacker, "Vienna RNA secondary structure server," Nucleic Acids Research, vol. 31, no. 13, pp. 3429-3431, 2003.

[34] M. Zuker, "Mfold web server for nucleic acid folding and hybridization prediction," Nucleic Acids Research, vol. 31, no. 13, pp. 3406-3415, 2003.

[35] A. Y. Ogurtsov, S. A. Shabalina, A. S. Kondrashov, and M. A. Roytberg, "Analysis of internal loops within the RNA secondary structure in almost quadratic time," Bioinformatics, vol. 22, no. 11, pp. 1317-1324, 2006.

[36] E. Rivas and S. R. Eddy, "Secondary structure alone is generally not statistically significant for the detection of noncoding RNAs," Bioinformatics, vol. 16, no. 7, pp. 583-605, 2000.

[37] T. T. Tran, F. Zhou, S. Marshburn, M. Stead, S. R. Kushner, and Y. Xu, "De novo computational prediction of non-coding RNA genes in prokaryotic genomes," Bioinformatics, vol. 25, no. 22, pp. 2897-2905, 2009.

[38] S. Agarwal, C. Vaz, A. Bhattacharya, and A. Srinivasan, "Prediction of novel precursor miRNAs using a context-sensitive hidden Markov model (CSHMM)," BMC Bioinformatics, vol. 11, supplement 1, article S29, 2010.

[39] D. Gautheret and A. Lambert, "Direct RNA motif definition and identification from multiple sequence alignments using secondary structure profiles," Journal of Molecular Biology, vol. 313, no. 5, pp. 1003-1011, 2001.

[40] J. S. Pedersen, G. Bejerano, A. Siepel et al., "Identification and classification of conserved RNA secondary structures in the human genome," PLoS Computational Biology, vol. 2, no. 4, article e33, 2006.
[41] Y. Ding, C. Y. Chan, and C. E. Lawrence, "Sfold web server for statistical folding and rational design of nucleic acids," Nucleic Acids Research, vol. 32, pp. W135-W141, 2004.

[42] R. Nussinov and A. B. Jacobson, "Fast algorithm for predicting the secondary structure of single-stranded RNA," Proceedings of the National Academy of Sciences of the United States of America, vol. 77, no. 11, pp. 6903-6913, 1980.

[43] J. S. McCaskill, "The equilibrium partition function and base pair binding probabilities for RNA secondary structure," Biopolymers, vol. 29, no. 6-7, pp. 1105-1119, 1990.

[44] B. H. Zhang, X. P. Pan, S. B. Cox, G. P. Cobb, and T. A. Anderson, "Evidence that miRNAs are different from other RNAs," Cellular and Molecular Life Sciences, vol. 63, no. 2, pp. 246-254, 2006.

[45] Y. Gupta, M. Witte, S. Möller et al., "PtRNApred: computational identification and classification of post-transcriptional RNA," Nucleic Acids Research, vol. 42, no. 22, article e167, 2014.

[46] Z. J. Lu, K. Y. Yip, G. Wang et al., "Prediction and characterization of noncoding RNAs in C. elegans by integrating conservation, secondary structure, and high-throughput sequencing and array data," Genome Research, vol. 21, no. 2, pp. 276-285, 2011.

[47] J. Hackermüller, K. Reiche, C. Otto et al., "Cell cycle, oncogenic and tumor suppressor pathways regulate numerous long and macro non-protein-coding RNAs," Genome Biology, vol. 15, no. 3, article R48, 2014.

[48] N. Philippe, E. Bou Samra, A. Boureux et al., "Combining DGE and RNA-sequencing data to identify new polyA+ non-coding transcripts in the human genome," Nucleic Acids Research, vol. 42, no. 5, pp. 2820-2832, 2014.

[49] P. Videm, D. Rose, F. Costa, and R. Backofen, "BlockClust: efficient clustering and classification of non-coding RNAs from short read RNA-seq profiles," Bioinformatics, vol. 30, no. 12, pp. I274-I282, 2014.

[50] P. Gellert, Y. Ponomareva, T. Braun, and S. Uchida, "Noncoder: a web interface for exon array-based detection of long noncoding RNAs," Nucleic Acids Research, vol. 41, no. 1, article e20, 2013.

[51] Z. Lu and A. G. Matera, "Vicinal: a method for the determination of ncRNA ends using chimeric reads from RNA-seq experiments," Nucleic Acids Research, vol. 42, no. 9, article e79, 2014.

[52] Y. Y. Leung, P. Ryvkin, L. H. Ungar, B. D. Gregory, and L.-S. Wang, "CoRAL: predicting non-coding RNAs from small RNAsequencing data," Nucleic Acids Research, vol. 41, no. 14, article e137, 2013.

[53] Y. Hoogstrate, G. Jenster, and E. S. Martens-Uzunova, "FlaiMapper: computational annotation of small ncRNA-derived fragments using RNA-seq high-throughput data, Bioinformatics, vol. 31, no. 5, pp. 665-673, 2015.

[54] P. Jiang, H. Wu, W. Wang, W. Ma, X. Sun, and Z. Lu, "MiPred: classification of real and pseudo microRNA precursors using random forest prediction model with combined features," Nucleic Acids Research, vol. 35, no. 2, pp. W339-W344, 2007.

[55] P. Xuan, M. Guo, X. Liu, Y. Huang, W. Li, and Y. Huang, "PlantMiRNAPred: efficient classification of real and pseudo plant pre-miRNAs," Bioinformatics, vol. 27, no. 10, pp. 13681376, 2011.

[56] T. B. Hansen, M. T. Venø, J. Kjems, and C. K. Damgaard, "miRdentify: high stringency miRNA predictor identifies several novel animal miRNAs," Nucleic Acids Research, vol. 42, no. 16, article e124, 2014. 
[57] S. Tyagi, C. Vaz, V. Gupta et al., "CID-miRNA: a web server for prediction of novel miRNA precursors in human genome," Biochemical and Biophysical Research Communications, vol. 372, no. 4, pp. 831-834, 2008.

[58] Y. Xu, X. Zhou, and W. Zhang, "MicroRNA prediction with a novel ranking algorithm based on random walks," Bioinformatics, vol. 24, no. 13, pp. i50-i58, 2008.

[59] S. Moxon, F. Schwach, T. Dalmay, D. MacLean, D. J. Studholme, and V. Moulton, "A toolkit for analysing large-scale plant small RNA datasets," Bioinformatics, vol. 24, no. 19, pp. 2252-2253, 2008.

[60] E. Zhu, F. Zhao, G. Xu et al., "mirTools: microRNA profiling and discovery based on high-throughput sequencing," Nucleic Acids Research, vol. 38, no. 2, pp. W392-W397, 2010.

[61] M. Hackenberg, M. Sturm, D. Langenberger, J. M. FalcónPérez, and A. M. Aransay, "miRanalyzer: a microRNA detection and analysis tool for next-generation sequencing experiments," Nucleic Acids Research, vol. 37, no. 2, pp. W68-W76, 2009.

[62] G. Barturen, A. Rueda, M. Hamberg et al., "sRNAbench: profiling of small RNAs and its sequence variants in single or multi-species high-throughput experiments," Methods in Next Generation Sequencing, vol. 1, no. 1, pp. 21-31, 2014.

[63] D. Managadze, A. E. Lobkovsky, Y. I. Wolf, S. A. Shabalina, I. B. Rogozin, and E. V. Koonin, "The vast, conserved mammalian lincRNome," PLoS Computational Biology, vol. 9, no. 2, Article ID e1002917, 2013.

[64] G. V. Glazko, B. L. Zybailov, and I. B. Rogozin, "Computational prediction of polycomb-associated long non-coding RNAs," PLoS ONE, vol. 7, no. 9, Article ID e44878, 2012.

[65] S. Boerner and K. M. McGinnis, "Computational identification and functional predictions of long noncoding RNA in Zea mays," PLoS ONE, vol. 7, no. 8, Article ID e43047, 2012.

[66] T. Derrien, R. Johnson, G. Bussotti et al., “The GENCODE v7 catalog of human long noncoding RNAs: analysis of their gene structure, evolution, and expression," Genome Research, vol. 22, no. 9, pp. 1775-1789, 2012.

[67] M. Guttman, I. Amit, M. Garber et al., "Chromatin signature reveals over a thousand highly conserved large non-coding RNAs in mammals," Nature, vol. 458, no. 7235, pp. 223-227, 2009.

[68] Y. Saeys, I. Inza, and P. Larrañaga, "A review of feature selection techniques in bioinformatics," Bioinformatics, vol. 23, no. 19, pp. 2507-2517, 2007.

[69] T.-T. Liu, D. Zhu, W. Chen et al., "A global identification and analysis of small nucleolar RNAs and possible intermediatesized non-coding RNAs in or," Molecular Plant, vol. 6, no. 3, pp. 830-846, 2013.

[70] X. Wang, J. Zhang, F. Li et al., "MicroRNA identification based on sequence and structure alignment," Bioinformatics, vol. 21, no. 18, pp. 3610-3614, 2005.

[71] K. M. Chisholm, Y. Wan, R. Li, K. D. Montgomery, H. Y. Chang, and R. B. West, "Detection of long non-coding RNA in archival tissue: correlation with polycomb protein expression in primary and metastatic breast carcinoma," PLoS ONE, vol. 7, no. 10, Article ID e47998, 2012.

[72] X. C. Quek, D. W. Thomson, J. L. V. Maag et al., "IncRNAdb v2.0: expanding the reference database for functional long noncoding RNAs," Nucleic Acids Research, vol. 43, no. 1, pp. D168-D173, 2015.

[73] J. Ponjavic, P. L. Oliver, G. Lunter, and C. P. Ponting, "Genomic and transcriptional co-localization of protein-coding and long non-coding RNA pairs in the developing brain," PLoS Genetics, vol. 5, no. 8, Article ID e1000617, 2009.

[74] T. R. Mercer, M. E. Dinger, and J. S. Mattick, "Long non-coding RNAs: insights into functions," Nature Reviews Genetics, vol. 10, no. 3, pp. 155-159, 2009.

[75] D. Rose, M. Hiller, K. Schutt, J. Hackermüller, R. Backofen, and P. F. Stadler, "Computational discovery of human coding and non-coding transcripts with conserved splice sites," Bioinformatics, vol. 27, no. 14, pp. 1894-1900, 2011.

[76] Q. Zou, J. Guo, Y. Ju, M. Wu, X. Zeng, and Z. Hong, "Improving tRNAscan-SE annotation results via ensemble classifiers," Molecular Informatics, vol. 34, no. 11-12, pp. 761-770, 2015.

[77] T. M. Lowe and S. R. Eddy, "TRNAscan-SE: a program for improved detection of transfer RNA genes in genomic sequence," Nucleic Acids Research, vol. 25, no. 5, pp. 955-964, 1997.

[78] P. P. Chan and T. M. Lowe, "GtRNAdb: a database of transfer RNA genes detected in genomic sequence," Nucleic Acids Research, vol. 37, no. 1, pp. D93-D97, 2009.

[79] J. Hertel, I. L. Hofacker, and P. F. Stadler, "SnoReport: computational identification of snoRNAs with unknown targets," Bioinformatics, vol. 24, no. 2, pp. 158-164, 2008.

[80] P. Schattner, A. N. Brooks, and T. M. Lowe, "The tRNAscanSE, snoscan and snoGPS web servers for the detection of tRNAs and snoRNAs," Nucleic Acids Research, vol. 33, no. 2, pp. W686W689, 2005.

[81] L. Lestrade and M. J. Weber, "snoRNA-LBME-db, a comprehensive database of human H/ACA and C/D box snoRNAs," Nucleic Acids Research, vol. 34, pp. D158-D162, 2006.

[82] K. Lagesen, P. Hallin, E. A. Rødland, H.-H. Stærfeldt, T. Rognes, and D. W. Ussery, "RNAmmer: consistent and rapid annotation of ribosomal RNA genes," Nucleic Acids Research, vol. 35, no. 9, pp. 3100-3108, 2007.

[83] D. Betel, R. Sheridan, D. S. Marks, and C. Sander, "Computational analysis of mouse piRNA sequence and biogenesis," PLoS Computational Biology, vol. 3, no. 11, article e222, 2007.

[84] Y. Zhang, X. Wang, and L. Kang, "A k-mer scheme to predict piRNAs and characterize locust piRNAs," Bioinformatics, vol. 27, no. 6, pp. 771-776, 2011.

[85] Q. Zou, J. Li, Q. Hong et al., "Prediction of microRNA-disease associations based on social network analysis methods," BioMed Research International, vol. 2015, Article ID 810514, 9 pages, 2015.

[86] Q. Zou, J. Li, L. Song, X. Zeng, and G. Wang, "Similarity computation strategies in the microRNA-disease network: a survey," Briefings in Functional Genomics, vol. 15, no. 1, pp. 5564, 2016.

[87] T. Kin, K. Yamada, G. Terai et al., "fRNAdb: a platform for mining/annotating functional RNA candidates from noncoding RNA sequences," Nucleic Acids Research, vol. 35, no. 1, pp. D145-D148, 2007.

[88] C. Liu, B. Bai, G. Skogerbø et al., "NONCODE: an integrated knowledge database of non-coding RNAs," Nucleic Acids Research, vol. 33, pp. D112-D115, 2005.

[89] T. D. Wu and C. K. Watanabe, "GMAP: a genomic mapping and alignment program for mRNA and EST sequences," Bioinformatics, vol. 21, no. 9, pp. 1859-1875, 2005.

[90] B. Langmead, C. Trapnell, M. Pop, and S. L. Salzberg, "Ultrafast and memory-efficient alignment of short DNA sequences to the human genome," Genome Biology, vol. 10, no. 3, article R25, 2009. 
[91] D. Sharon, H. Tilgner, F. Grubert, and M. Snyder, "A singlemolecule long-read survey of the human transcriptome," Nature Biotechnology, vol. 31, no. 11, pp. 1009-1014, 2013.

[92] S. Li, S. W. Tighe, C. M. Nicolet et al., "Erratum: multiplatform assessment of transcriptome profiling using RNAseq in the ABRF next-generation sequencing study," Nature Biotechnology, vol. 32, no. 11, p. 1166, 2014. 

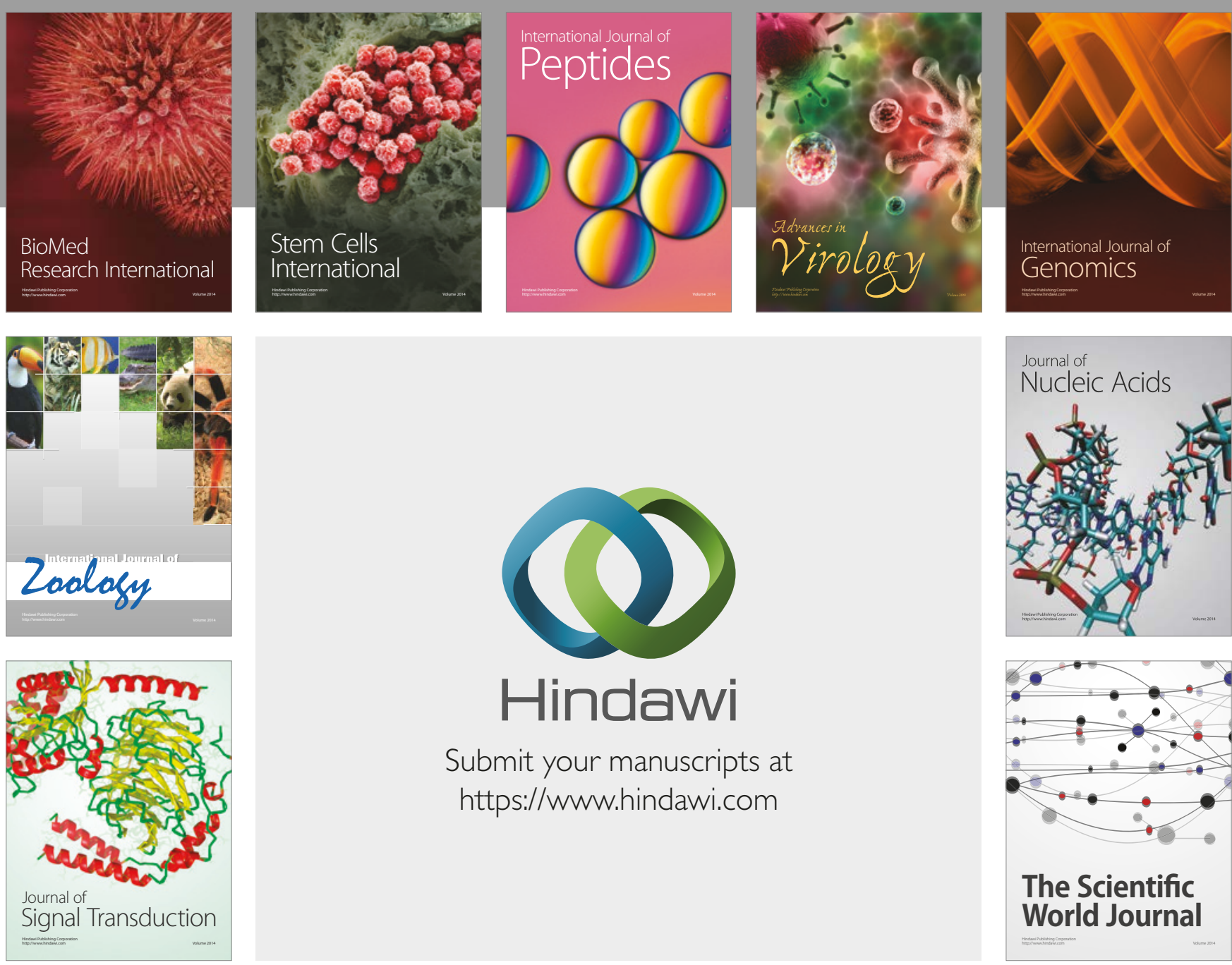

Submit your manuscripts at

https://www.hindawi.com
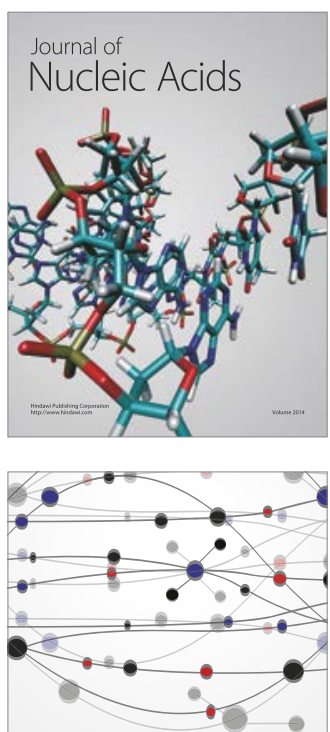

The Scientific World Journal

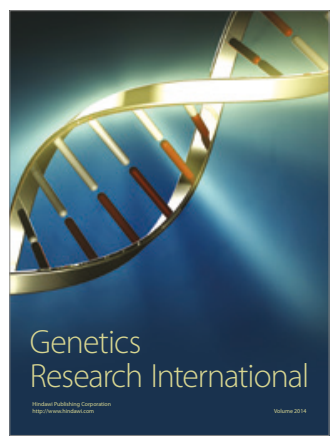

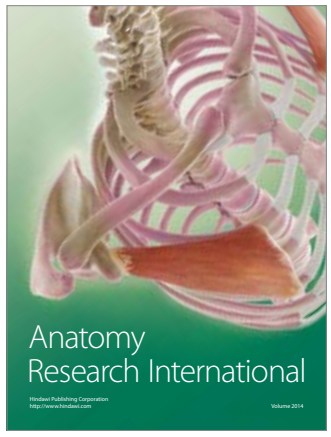

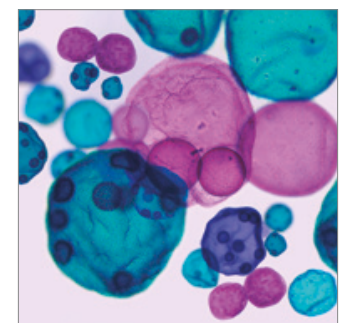

International Journal of Microbiology
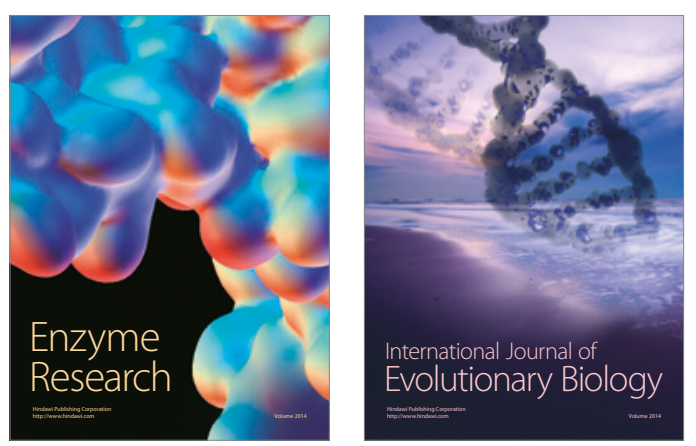
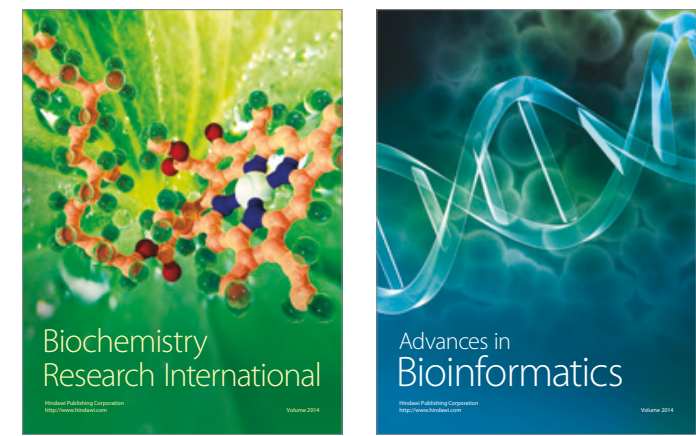

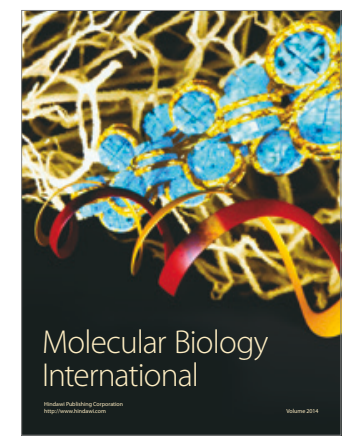

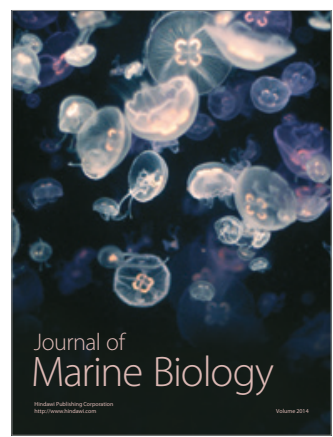

